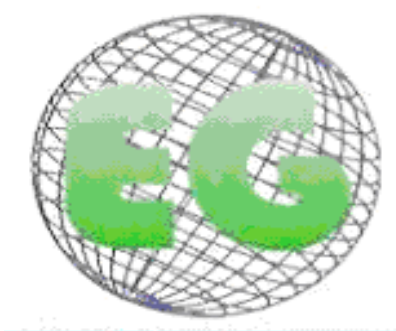

ISSN 1695-6141

$\mathrm{N}^{\circ} 24$
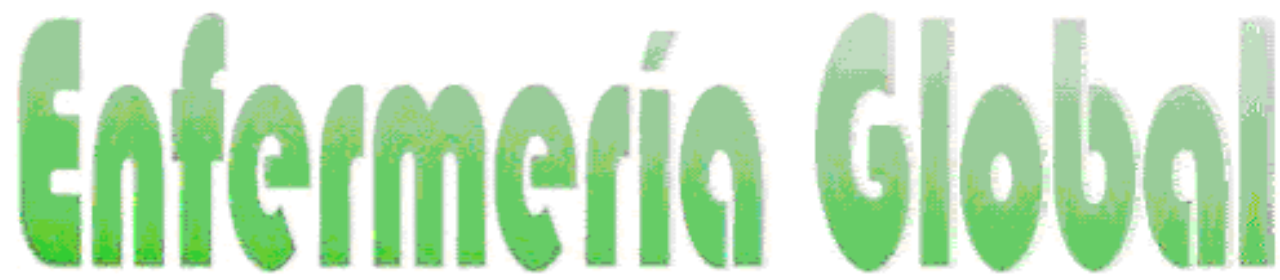

Revista electrónica trimestral de Enfermería

Octubre 2011

www.um.es/egloball

\title{
Detección y atención a la violencia de género en el embarazo. Una asignatura pendiente
}

Detecting and Attending to Gender Violence during Pregnancy. An unresolved matter

*Martínez Galiano, Juan Miguel

*Matrón. Servicio de Paritorio. Hospital San Juan de la Cruz. Úbeda. Jaén.

Palabras clave: violencia de género; Embarazo; Maltrato contra las mujeres; Personal sanitario.

Keywords: gender violence; pregnancy; maltreatment of women; Health workers

\section{RESUMEN}

Objetivos: Determinar el número de casos de violencia de género (VG) que el personal sanitario encargado del control de embarazo era consciente que se habia atendido. Identificar la existencia o no de recursos de los que dispone este personal para abordar este problema. Conocer la prioridad que este personal asigna a la VG dentro del sistema sanitario.

Método: estudio observacional descriptivo, sujetos de estudio: personal sanitario con funciones en el control del embarazo. Se utilizó un cuestionario de elaboración propia. Se realizó un análisis descriptivo de los datos. Resultados: El 93,10\% (81) creen que son menos de diez al año las mujeres atendidas El 95,40\% (83) afirma que no tiene los recursos necesarios para poder actuar Se otorga una media de 8,14 $\pm 1,45$ sobre 10 en el orden de priorización.

Conclusión: El personal parece estar sensibilizado aunque no se considera preparado para abordar el problema.

\section{ABSTRACT}

Aims: to determine the number of cases of gender violence (GV) that health personnel in charge of pregnancy control were aware they had attended to; to identify whether resources were available to the personnel to address this problem; to ascertain the priority these personnel assign to GV within the health system.

Method: descriptive, observational study. Subjects were health personnel working in pregnancy control. A questionnaire developed by the researchers was used. Descriptive analysis of the data was made.

Results: $93.10 \%$ (81) believed that they attended fewer than ten such cases a year. $95.40 \%(83)$ stated that they did not have the necessary resources to enable them to act. A mean score of $8,14 \pm 1,45$ out of 10 was awarded regarding prioritization. 
Conclusion: The personnel seemed to be aware of the problem but did not consider themselves prepared to address it.

\section{INTRODUCCIÓN}

Todo acto de violencia basado en la pertenencia al sexo femenino que tenga o pueda tener como resultado un daño o sufrimiento físico, sexual o psicológico para la mujer, así como las amenazas de tales actos, la coacción o la privación arbitraria de la libertad, tanto si se producen en la vida pública como en la vida privada es considerado violencia de género (VG). ${ }^{1}$

A pesar de la alta prevalencia (20\%-75\%) de la VG y las consecuencias a nivel de salud física, psíquica y social que de esta se derivan hacia la mujer, el personal sanitario no lo reconoce como tal problema. ${ }^{2}$ El papel de los servicios sanitarios es fundamental para el abordaje de este tipo de violencia pero sólo se identifica un $1 \%$ del total estimado de casos de mujeres maltratadas. ${ }^{3}$

La evidencia cientifica reconoce el embarazo como una situación de mayor vulnerabilidad para sufrir VG, las consecuencias derivadas de este maltrato sufrido durante el embarazo tiene repercusiones a corto, a medio y a largo plazo. Supone un gasto sanitario a la administración y las mujeres que sufren VG tiene mayor riesgo de sufrir estrés, depresión, abuso de drogas, alcohol y tabaco, pueden sufir más abortos, más complicaciones postparto, más infecciones, mala alimentación, anemia y metrorragias, lo que va a repercutir en la evolución del embarazo y en el desarrollo del feto; estas mujeres acuden más a los centros sanitarios y también se realizan más hospitalizaciones que no tienen como causa algo relacionado con el embarazo. Los recién nacidos de madres que sufren VG suelen tener más riesgo de bajo peso al nacer y de parto pretérmino y estos niños y niñas en el futuro pueden ser más violentos y tienen mayor riesgo de morir antes de los cinco años. ${ }^{4}$

La Asociación de Ginecólogos y Obstetras Americanos (ACOG), recomienda efectuar una búsqueda activa de casos de violencia en el embarazo. ${ }^{5}$ Actualmente la Sociedad Española de Ginecología y Obstetricia y la Federación de Asociaciones de Matronas de España (FAME) no tienen ningún protocolo respecto al tema de VG. El proceso de control del embarazo de la Consejería de Salud de la Junta de Andalucía y en el protocolo común para la actuación sanitaria ante la violencia de género de la Comisión Contra la Violencia de Género del Consejo Interterritorial del Sistema Nacional de Salud no contemplan medidas a llevar a cabo durante el control del embarazo para la detección y abordaje de este problema $^{1,6}$

Ante el papel fundamental que puede desarrollar el personal sanitario y los servicios de salud para la erradicación de la VG y la mayor vulnerabilidad del embarazo, así como la falta de medidas para la detección de casos, nos propusimos conocer la actitud y sensibilización de este personal encargado del control del embarazo ante la VG para en un futuro poder contemplar la implementación de medidas; así nos planteamos los siguientes objetivos:

- Determinar el número de casos de VG que el personal sanitario encargado del control embarazo era consciente que se habian atendido.

- Identificar la existencia o no de recursos de los que dispone el personal sanitario encargado del control del embarazo para abordar el tema de VG. 
- Conocer la prioridad que el personal sanitario encargado del control del embarazo asigna al problema de la VG dentro del sistema sanitario.

\section{METODOLOGÍA}

Se diseñó un estudio observacional descriptivo cuyos sujetos de estudio eran matronas/matrones y ginecólogos/ginecólogas de la Comunidad Autónoma de Andalucía, es decir, personal sanitario con funciones en el control del embarazo. Se utilizó un cuestionario de elaboración propia no válidado en el cual se encontraban los items necesarios para recoger la información que se pretendía en nuestros objetivos y que se desprende de las variables que se tuvieron en cuenta y que se pueden ver en la Tabla 1. Se enviaron durante el mes de abril del 2010 vía correo electrónico 117 mails con el cuestionario a matronas/matrones y 39 a ginecólogos/ginecologas solicitándoles su colaboración con la autocumplimentación del cuestionario y el posterior reenvío a la dirección de la que procedía antes del 30 de junio del 2010. Posteriormente, se realizó un análisis descriptivo de los datos. Las variables continuas se expresaron como media y desviación típica, y las categóricas como frecuencias absolutas (n) y relativas (\%). Se utilizó el programa Epidat 3.1.

Tabla 1. Variables de estudio

-Categoria Profesional: Matrón/a, Ginecólogo/a, Otras.

-Edad

-Sexo: Hombre, Mujer

-Ámbito de trabajo: Atención primaria, Atención especializada, Ambas.

-Años de experiencia profesional en la categoria: <5 años, Entre 5 y 15 años, $>15$ años.

-Consideración del maltrato a las mujeres como un problema de salud: Sí, No, No sabe.

- Posibilidad de llevar a cabo medidas desde su puesto de trabajo orientadas a la erradicación del maltrato contra las mujeres: Sí, No, No sabe.

-Atención a mujeres en situación de maltrato a lo largo de la vida profesional: Sí, No.

- Total de mujeres en situación de maltrato que pueden ser atendidas en el control del embarazo: Ninguna, <10 mujeres/año, Entre 10-50 mujeres/año, $>50$ mujeres/año.

-Consideración que el tema del matrato sea tema prioritario para la administración sanitaria: Sí, No, No sabe.

-Puntuación de 0 a 10, donde 0 es nada y 10 máxima prioridad, el orden de importancia que debería otorgarle la administración al tema del maltrato y así priorizar los recursos a asignarle.

-Formación del personal en temas de violencia de género/maltrato: Sí, No.

-Necesidad de formación en tema de maltrato contra las mujeres a los profesionales sanitarios: Sí, No, No sabe.

-Disponibilidad por parte del personal de los recursos necesarios (formación y recursos materiales) para poder detectar y atender a las mujeres en situación de maltrato: Sí, No, No sabe.

-Factor de riesgo más importante que tiene la mujer para sufrir malos tratos: Nivel socioeconómicoy cultural bajo, Ser Mujer, Inmigración, Tener dependecia afectiva del matrador, Otros; Especificar:

-Necesidad de implementar medidas para la detección de casos de malos tratos en mujeres durante el control del embarazo: Sí, No, Antes hay que hacer otras cosas, No sabe. 


\section{RESULTADOS}

Se obtuvieron 87 cuestionarios cumplimentados, de estos el $88,50 \%$ (77) correspondían a matronas/matrones, el 10,35\% (9) a medicina especialista en obstetricia y ginecología y el $1,15 \%$ (1) no identificó su categoria profesional.

La edad media del personal que respondió al cuestionario era de $32,75 \pm 5,91$ años. El $6,90 \%(6)$ desarrolla su labor profesional en atención primaria y el $93,10 \%(81)$ en atención especializada, el $9,20 \%$ (8) son hombres y el $90,80 \%$ (79) son mujeres con una experiencia profesional en la categoría de menos de cinco años el $72,41 \%$ (63), de entre cinco y quince años el $24,14 \%(21)$ y de más de quince años el 3,45\% (3).

El 1,13\% (1) no sabía si el maltrato era un problema de salud, el 3,45\% (3) no consideró que el maltrato fuese un problema de salud y el $95,40 \%$ (83) afirmaba que el maltrato era un problema de salud. El $82,76 \%$ (75) cree que desde su labor profesional diaria puede contribuir a la erradicación de la violencia de género, el 4,60\% (4) no lo sabe y el 9,20\% (8) cree que no.

Según el 6,90\% (6) de estos profesionales entre diez y cincuenta mujeres del total de las que son atendidas durante el control del embarazo y/o parto son mujeres en situación de maltrato, el $93,10 \%$ (81) creen que son menos de diez al año las mujeres en esta situación. El $91,96 \%$ (80) ha atendido alguna vez a lo largo de su carrera profesional alguna mujer en situación de maltrato.

El 87,36\% (76) tenia algún tipo de formación en temas de VG. El 95,40\% (83) afirma que no tiene los recursos necesarios para poder actuar correctamente en la atención prestada a las mujeres en situación de maltrato. El 97,70\% (85) ve necesaria la formación en temas de VG.

La media que le otorga al problema del maltrato este personal sanitario para que la administración sanitaria priorice la asignación de recursos es de $8,14 \pm 1,45$ sobre una puntuación máxima de 10. El factor de riesgo más importante para sufrir maltrato según el personal sanitario se encuentra reflejado en el Gráfico 1.

\section{Gráfico 1. Factores de riesgo para ser mujer maltratada, según el personal sanitario}

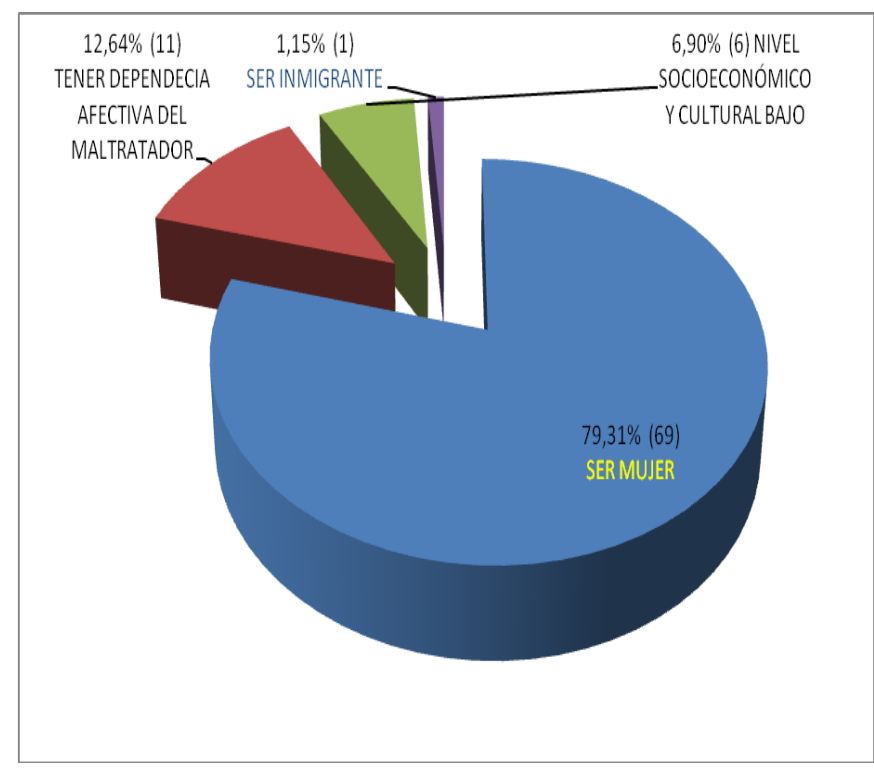


El 94,25\% (82) cree que se deberían de poner medidas para la detección de los casos de maltrato durante el control rutinario del embarazo, el $2,30 \%$ (2) no saben y el $3,45 \%$ (3) creen que antes hay que hacer otras cosas.

\section{DISCUSIÓN}

Entre las limitaciones del estudio destacar que de existir un sesgo de selección asociado a la no respuesta, éste habrá tenido una influencia mínima sobre la validez de los resultados pues no existen a priori razones para pensar que el personal que respondió difiera sustancialmente del que no respondió. Al tratarse de un cuestionario autoadministrado, elegido por llegar al mayor número de sujetos, es posible que la diferente interpretación de las preguntas y respuestas por parte del personal haya podido introducir un sesgo de clasificación, que en cualquier caso no consideramos que afecte de manera importante a los resultados.

El número de casos detectados y/o atendidos en un año por la mayoría del personal está muy por debajo de los casos que encontró en el estudio de Gómez et al ${ }^{7}$ aunque esta cifra va en consonancia con otros resultados ${ }^{3,8}$ como los del estudio de Valdez-Santiago en el cual los médicos señalaron que la violencia durante el embarazo era una situación poco frecuente en la población que atienden.

La mayor parte manifiesta que poseen formación en temas de maltrato en contra de lo que se han obtenido en otros estudios ${ }^{9}$ pero también admiten que no disponen de los recursos necesarios para hacer frente al problema, puede que por ello asignen un alto grado de prioridad porque la detección y actuación de dichos casos debe de realizarse con los métodos y formación adecuada. ${ }^{3,} 10$ Esta prioridad y la necesidad de formación que demandan nos informa de la sensibilización del personal en el tema coincidiendo con otros estudios. ${ }^{3,9}$ Además queda tambien patente su implicación en el tema ya que son receptivos y ven necesario la implentación de medidas que ellos mismos pueden llevar a cabo para la detección y actuación en casos de VG durante el control del embarazo.

Alrededor del $80 \%$ consideran como principal factor de riesgo para sufrir maltrato el hecho de ser mujer ${ }^{1}$ considerando que cualquier mujer puede estar en situación de maltrato. Al contrario que otros estudios este personal si considera la VG como un problema de salud. ${ }^{3}$

El diagnóstico y la atención a la VG están incluidos en el Real Decreto 1030/2006, de 15 de septiembre $^{1}$, el personal sanitario parece estar sensibilizado y responsabilizado aunque no se considera preparado para abordar el problema, la formación parece una pieza fundamental para superar este obstáculo. 3, 4,9

\section{CONCLUSIÓN}

El personal sanitario encargado del control del embarazo, parto y puerperio infradiagnostica los casos de violencia doméstica.

Este personal concede una alta prioridad al problema pero no dispone de los recursos necesarios para hacerle frente.

La respuesta sanitaria no debe sólo estar basada en la sensibilidad, sino también en la responsabilidad, en VG no se puede adoptar «una posición neutral», puesto que ello conllevaría una permanencia de la misma. 


\section{BIBLIOGRAFÍA}

1- Ministerio de Sanidad y Consumo. Protocolo común para la actuación sanitaria ante la violencia de género. Madrid. Ministerio de Sanidad y Consumo, 2007.

2-Valdez-Santiago R, Sanín-Aguirre $L$ H. La violencia doméstica durante el embarazo y su relación con el peso al nacer. Salud Pública de Méx 1996; 38:352-362

3- Coll-Vinent B, Echeverría T, Farràs U, Rodríguez D, Millá J, Santiñà M. El personal sanitario no percibe la violencia doméstica como un problema de salud. Gac Sanit. 2008; 22(1):7-10

4-Velasco Juez MC. Recomendaciones para la detección precoz de la violencia en el embarazo. Matronas Prof. 2008; 9 (3): 32-37

5- American College of Obstetricians and Gynecologists and Centers for Disease Control and Prevention. Work Group on the Prevention of Violence during Pregnancy. Intimate partner violence during pregnancy. A guide for clinicians. Washington: ACOG \& CDC, 2000. Consultado el 6 de Octubre de 2009. Disponible en: http://www.cdc.gov/reproductivehealth/violence/IntimatePartnerViolence/index.htm

6-Junta de Andalucía. Consejería de Salud. Proceso Asistencial Integrado. Embarazo, Parto y Puerperio. 2a Ed. Sevilla 2005

7- Gómez MC, Dorado MI, Bedoya Bergua JA, Cayuela A, Alonso MJ, Hidalgo D. Violencia contra la mujer. Resultados de una encuesta hospitalaria. Prog Obstet Ginecol. 2004; 47(11): $511-520$

8-Valdez-Santiago R, Arenas-Monreal L, Hernández-Tezoquipa I. Experiencia de las parteras en la identificación de mujeres maltratadas durante el embarazo. Salud Pública Mex 2004; 46:56-63.

9- Robledo Martín J, de la Fuente Aparicio D, Salamanca Castro AB, Sánchez Castro S. Percepción de las mujeres maltratadas sobre la atención sanitaria recibida. Nure Investigación, no 20, Enero-Febrero 06

10- Plazaola-Castaño Juncal, Ruiz-Pérez Isabel, Hernández-Torres Elisa. Validación de la versión corta del Woman Abuse Screening Tool para su uso en atención primaria en España. Gac Sanit.2008; 22(5): 415-420. 